\title{
Sostenibilidad Curricular: Una Mirada desde las Aportaciones del Profesorado de la Universidad de Málaga
}

\author{
Curricular Sustainability: A View from the Contributions of \\ Teaching Staff at the University of Málaga
}

\author{
María José Alcalá del Olmo ${ }^{1 *}$ \\ María Jesús Santos 2 \\ Juan José Leiva ${ }^{1}$ \\ Antonio Matas ${ }^{1}$ \\ ${ }^{1}$ Universidad de Málaga, España \\ ${ }^{2}$ Universidad Internacional de La Rioja, España
}

\begin{abstract}
El propósito de este estudio ha sido conocer las percepciones y competencias de los profesionales de la educación de la Universidad de Málaga en la puesta en práctica de la Educación para el Desarrollo Sostenible. Siguiendo una metodología cualitativa, se han diseñado entrevistas semiestructuradas ad hoc fundamentadas en un paradigma interpretativo. La muestra ha estado constituida por docentes del Grado en Educación Primaria y Educación Social de la Universidad de Málaga. Las cuestiones abordadas han permitido valorar en qué medida el profesorado ha integrado el discurso de la sostenibilidad en su rol profesional, junto a las relaciones establecidas entre la universidad y la necesidad de trabajar una educación comprometida con la mejora y el respeto al entorno. De los resultados se desprenden ciertas dificultades para trabajar sistemáticamente la sostenibilidad, originadas por la carencia de una formación específica, aunque han podido apreciarse actitudes favorables en el profesorado para su integración transversal, bien sea buscando conexiones epistemológicas o enfatizando un mayor protagonismo a la adquisición de competencias prácticas en el proceso de aprendizaje del alumnado. En la misma línea que otros estudios, queda patente la relevancia otorgada al trabajo interdisciplinar, la formación permanente y la investigación.
\end{abstract}

Descriptores: Educación superior; Sostenibilidad curricular; Profesor; Competencias; Educación para el desarrollo sostenible.

\begin{abstract}
The purpose of this study has been to know the perceptions and competencies of professionals in education at the University of Malaga in the implementation of the Education for Sustainable Development. Following a qualitative methodology, ad hoc semi-structured interviews have been designed based on an interpretative paradigm. The sample has been made up of teachers corresponding to the Degree in Primary Education and Social Education of the University of Malaga. The questions addressed have allowed us to assess the extent to which university professors have integrated the speech of the sustainability on their professional role, as well as the relationships between the university and the need to work an education committed to improving and respecting the environment. Among some of the results obtained, the existence of difficulties in systematically working on sustainability stands out, due to the lack of specific training. Favourable attitudes have been observed in the teaching staff towards introducing changes in their practice aimed at the transversal integration of sustainability, either looking for epistemological links or connections or, where appropriate, emphasizing greater prominence to the acquisition of practical skills in the student learning process. As with other studies, it is clear the importance of interdisciplinary work, permanent training and a predisposition to research.
\end{abstract}

Keywords: Higher education; Curricular sustainability; Teacher; Competencies; Education for sustainable development.

*Contacto: mjalcaladelolmo@uma.es

ISSN: 2254-3139

www.rinace.net/riejs/

revistas.uam.es/riejs
Recibido: $\quad 11$ de noviembre 2019

$1^{\text {a }}$ Evaluación: 25 de marzo 2020

$2^{\text {a }}$ Evaluación: 27 de abril 2020

Aceptado: 15 de mayo 2020 


\section{Introducción}

Los conflictos ecológicos forman parte de nuestra realidad. Todo ello deja sentir sus influencias en los contextos educativos, lo que hace necesaria una educación comprometida con el respeto al Planeta en todos los niveles formativos, incluido el universitario.

Esta temática ha resultado de interés en la comunidad científica en los últimos años, siendo profusos los estudios en los que se revela la importancia de que la universidad asuma la responsabilidad de promover una educación comprometida en la defensa y el respeto hacia el medio ambiente (Alba, 2017; Antúnez, Gomera y Villamandos, 2017; Aznar et al., 2014; Collado, 2017; Geli de Ciurana, Collazo y Mulá, 2019; Martínez-Lirola, 2018; MurgaMenoyo, 2018; Murga-Menoyo y Novo, 2017; Novo y Murga-Menoyo, 2015; Villamandos, Gomera y Antúnez, 2019).

En este escenario puede enmarcarse la Educación Ambiental, como herramienta pedagógica destinada a promover competencias para la acción, que reviertan en una mejora de las relaciones establecidas entre el individuo y su entorno, con el fin de ofrecer lo mejor del mismo a las generaciones del futuro, siendo así pilar prioritario a favor del desarrollo sostenible (Gil y Vilches, 2017; UNESCO, 2012).

La Educación Ambiental cuenta con una dilatada trayectoria histórica, adquiriendo gran relevancia con el transcurso del tiempo las dimensiones social, ambiental y educativa, colonizando el término de desarrollo sostenible el marco de la misma (Jiménez-Fontana y García-González, 2017), para asumir respuestas coherentes que lleven a la humanidad al respeto y la defensa medioambiental.

En este sentido, puede aceptarse que la Educación Ambiental y la Educación para el Desarrollo Sostenible forman parte de una misma filosofía educativa, buscando potenciar el desarrollo de una conciencia de ciudadanía global, que haga a los educandos responsables, críticos y solidarios en un desarrollo humano sostenible, generándose una conciencia planetaria que busca erradicar las desigualdades.

A lo largo de este trabajo, se ha optado por recurrir al término de Educación para el Desarrollo Sostenible, teniendo en cuenta la evolución epistemológica experimentada por la Educación Ambiental en las últimas décadas (Murga-Menoyo y Novo, 2017), siendo uno de sus propósitos principales educar para hacer realidad los principios vinculados con la sostenibilidad.

A todo ello se suma la importancia que en la actualidad se otorga a la educación como instrumento para contribuir a la resolución de la crisis planetaria, asumiendo, al mismo tiempo, que la fundamentación científica de la Educación para el Desarrollo Sostenible coincide con los argumentos que defienden una educación capaz de promover la justicia ambiental (Murga-Menoyo, 2018), comprometida en el camino que lleva a la transformación de nuestra sociedad.

Uno de los retos más significativos de las instituciones universitarias del siglo XXI es formar profesionales que no solo lleguen a ser capaces de actuar para promover un desarrollo humano más sostenible, sino que también sean críticos con el curso y la evolución que está tomando nuestra sociedad, cada vez más insostenible.

Pese a los esfuerzos realizados y aún dada la existencia de un gran respaldo institucional para hacer realidad una educación respetuosa con el Planeta en la universidad, son numerosas las investigaciones que, centradas en analizar el proceso de ambientalización 
curricular en la universidad, ponen de manifiesto tanto el largo camino que aún queda por recorrer, como la necesaria implicación del profesorado para generar cambios en la docencia acordes con el paradigma de la sostenibilidad (Albareda et al., 2017; Andrades, Larrán y Muriel de los Reyes, 2018; Leal-Filho et al., 2017; Segalás y Sánchez-Carracedo, 2019; Serrate et al., 2019; Valderrama et al., 2020).

Con la pretensión de conocer algunas dificultades que impiden el abordaje transversal de la sostenibilidad en la Educación Superior, Albareda y otros (2017) llevaron a cabo un estudio en el que participaron docentes de dos campus universitarios catalanes. La resistencia al cambio, la ausencia de una percepción sistémica en el abordaje de temas relacionados con el deterioro ecológico y la carencia de formación continua en Educación para el Desarrollo Sostenible fueron las principales barreras identificadas.

Andrades, Larrán y Muriel de los Reyes (2018) emprendieron un estudio en la Universidad de Cádiz para analizar el proceso de incorporación de la sostenibilidad en la estructura curricular universitaria. Además de verificar que el abordaje de la Educación para el Desarrollo Sostenible quedada limitado a disciplinas vinculadas a las ciencias ambientales, los autores pudieron constatar tanto la escasa formación del profesorado universitario en estas cuestiones, como la carencia de compromiso en iniciativas relacionadas con la sostenibilidad.

Leal Filho y otros (2017) realizaron una investigación en universidades distribuidas en diversas partes del mundo. Además de evidenciar la complejidad en la puesta en práctica de la Educación para el Desarrollo Sostenible en el marco universitario, los autores advierten el desconocimiento del profesorado sobre la forma de ambientalizar el currículum de su asignatura, con la consiguiente complejidad para trabajar de forma sistemática y estructurada aspectos relacionados con el medio ambiente en la Educación Superior.

Segalás y Sánchez-Carracedo (2019), por su parte, analizan el llamado Proyecto Edinsost, cuyo objetivo es valorar la implementación de la Educación para el Desarrollo Sostenible en las universidades españolas, para ofrecer a los estudiantes universitarios las competencias necesarias que permitan avanzar en la conformación de sociedades sostenibles. Estos autores, a partir del mencionado Proyecto, llevan a cabo, entre otras cuestiones, una valoración de la puesta en marcha de la Educación para el Desarrollo Sostenible por parte del docente universitario. Los resultados advierten la falta de conocimiento de las implicaciones de la sostenibilidad en la praxis profesional, cumpliendo la motivación un destacado papel, en tanto que son los profesores más motivados los que se esfuerzan en hacer realidad la sostenibilidad en las asignaturas en las que tienen asignada docencia.

Serrate y otros (2019) prosiguen analizando las percepciones de estudiantes universitarios, pertenecientes a los Grados de Pedagogía y Educación Social de la Universidad de Salamanca. Los resultados permiten vislumbrar que la formación en sostenibilidad resulta insuficiente, argumentando la necesidad de que el docente universitario reciba formación continua al respecto, para poder hacer realidad el abordaje interdisciplinar de la Educación para el Desarrollo Sostenible en el currículum universitario.

Valderrama y otros (2020) llevaron a cabo un estudio en la Universidad de Salamanca, Sevilla, Politécnica de Madrid e Internacional de Cataluña. Entre algunos de los 
principales hallazgos, cabe poner de relieve que el alumnado considera que la formación pedagógica que recibe en la universidad apenas se relaciona con la sostenibilidad, argumentando la necesidad de que el profesorado promueva la realización de trabajos de investigación y se esfuerce en relacionar los contenidos abordados en las asignaturas con la sostenibilidad, de cara a fomentar un aprendizaje más significativo y vivencial.

Teniendo en cuenta los argumentos anteriores y, con ellos, la importancia concedida al profesorado como agente catalizador del proceso de sostenibilidad curricular en la universidad, este estudio tiene como finalidad analizar las competencias y percepciones del profesorado de la Facultad de Educación de la Universidad de Málaga en la Educación para el Desarrollo Sostenible.

Para ello, se ha optado por una metodología cualitativa fundamentada en un paradigma de investigación interpretativo, relevante para estudiar, desde los valores, creencias y reflexiones de personas implicadas en contextos educativos concretos, una realidad que, como la educación para la sostenibilidad, se ha convertido en un desafío al que responder desde la Educación Superior.

\section{Educar para el desarrollo sostenible en el escenario universitario: Reto y necesidad}

La incorporación de los centros universitarios en el Espacio Europeo de Educación Superior llevó a la constitución de un nuevo enfoque generador de cambios sustanciales en la formación universitaria y la práctica docente, impulsando iniciativas con las que propiciar la adquisición de competencias, entre las que se incluyen las relacionadas con la sostenibilidad (Barth et al., 2007; López, León y Pérez, 2018; Murga-Menoyo, 2015; Murga-Menoyo y Novo, 2017).

Dichas competencias encuentran su razón de ser en la importancia de proporcionar una formación con la que conseguir profesionales críticos, dotados de instrumentos para actuar eficazmente ante los retos ecológicos actuales (UNESCO, 2017), siendo la educación un instrumento valioso para abogar por la justicia y la sostenibilidad, en un marco de alianzas entre justicia social y ambiental (Carneros, Murillo y Moreno-Medina, 2018; Derr, 2017).

Las directrices que emanan de dicho Espacio mencionan el desarrollo sostenible como una prioridad universitaria, buscando sinergias entre educación y sostenibilidad (Alba, 2017; Bautista y Díaz, 2017), para trabajar desde una óptica transversal cuestiones relativas a la degradación planetaria en el marco de todas las asignaturas y grados universitarios. Es así como surge el concepto de ambientalización curricular, entendido como un modo de incorporar en la formación superior aquellos aspectos que permitan desempeñar la profesión futura desde la ética y la responsabilidad hacia el desarrollo sostenible (Aleixo, Azeiteiro y Leal, 2017; Murga-Menoyo, 2017; Murga-Menoyo y Novo, 2017).

Desde estos planteamientos, dotar a la formación universitaria de un matiz ambiental no implica únicamente incorporar contenidos ambientales en las programaciones de las asignaturas, sino que supone integrar cambios globales en los procesos educativos, para promover una imagen amplia de la realidad, un pensamiento sistémico, un aprendizaje capaz de vincular acción con reflexión y una concepción holística de la educación, apostándose por garantizar un desarrollo integral de las facultades humanas.

Actualmente, educar para el desarrollo sostenible ocupa un lugar prioritario en la agenda educativa nacional e internacional (Murga-Menoyo, 2018). La UNESCO, en la 
Declaración de Aichi-Nagoya sobre la Educación para el Desarrollo Sostenible, concedería a la formación universitaria la responsabilidad de velar por la puesta en práctica de la sostenibilidad en todos sus marcos de actuación, contribuyendo a la conformación de sociedades sostenibles (UNESCO, 2014).

La Agenda 2030, relacionada con los Objetivos del Desarrollo Sostenible aprobados por la Asamblea General de las Naciones Unidas para el periodo 2016-2030 (ONU, 2015), supuso una apuesta por impulsar una formación comprometida con el respeto y defensa ambiental, vinculando la acción pedagógica con los principios de la sostenibilidad, para repercutir en el bienestar de las personas y la naturaleza (París, 2019).

Dichos Objetivos reflejan la necesidad del abordaje interdisciplinar de ciertas problemáticas sociales, entre las que están presentes, además de la sostenibilidad, las situaciones de vulnerabilidad y exclusión social (Guzmán y Ortiz, 2019), de las que debe responsabilizarse la formación superior.

En nuestro país, la Conferencia de Rectores de las Universidades Españolas (CRUE), aprobó un documento en 2005, revisado posteriormente en el año 2011, titulado Directrices para la Sostenibilidad Curricular (CRUE, 2012). Este fue remitido a las universidades para observar las estructuras curriculares desde una perspectiva sostenible, buscando asegurar la inclusión en todas las titulaciones universitarias de contenidos transversales con los que garantizar la promoción de competencias acordes con los valores de una educación respetuosa con el entorno (Aznar et al., 2017).

En el año 2018, dicha Conferencia diseñó un Plan de Acción para favorecer la transferencia de conocimientos en la universidad en coherencia con los objetivos delimitados en la Agenda 2030 para el Desarrollo Sostenible, en el horizonte temporal 2018-2020 (CRUE, 2018). De la misma forma, se puso de relieve que las carencias de las políticas de sostenibilidad en las universidades estaban relacionadas con el casi inexistente tratamiento transversal concedido a la Educación para el Desarrollo Sostenible, dependiendo su puesta en práctica del interés particular mostrado por algunos docentes sensibilizados en estas cuestiones.

Es por ello por lo que el interés residía en reforzar el compromiso de la comunidad universitaria en trabajar los principios del desarrollo sostenible en todas las titulaciones universitarias, analizando e impulsando la incorporación de competencias de sostenibilidad en la formación de estudiantes universitarios, personal docente e investigador y personal de administración y servicios.

Teniendo en cuenta los argumentos anteriores y subrayando el relevante rol del docente universitario en la conformación de estructuras curriculares sostenibles, los principales objetivos que sustentan la realización de este estudio son los siguientes: (i) analizar en qué medida el profesorado perteneciente a la Facultad de Ciencias de la Educación considera que la universidad actual debe comprometerse en hacer realidad la Educación para el Desarrollo Sostenible; (ii) indagar qué conocimiento presenta sobre esta enseñanza y sus funciones principales; (iii) conocer cuáles son las principales repercusiones que, a juicio del profesorado universitario, genera la sostenibilidad en su práctica profesional; (iv) clarificar qué competencias se consideran imprescindibles para trabajar la Educación para el Desarrollo Sostenible en la universidad; y (v) identificar hasta qué punto este sector profesional ha recibido formación para hacer realidad en las aulas el paradigma de la sostenibilidad. 


\section{Método}

De acuerdo con la naturaleza de los datos, en este estudio se ha seguido una metodología de corte cualitativo, fundamentada en un paradigma de investigación interpretativo con carácter inductivo, puesto que las competencias y percepciones del profesorado universitario han podido inducirse a partir del análisis de entrevistas realizadas. Todo ello ha permitido profundizar en algunas variables de estudio, reconocidas en las valoraciones y evidencias expresadas por el profesorado participante en la investigación.

La hipótesis de partida se relaciona con la existencia de dificultades ante las que se encuentra el profesorado universitario para trabajar la Educación para el Desarrollo Sostenible, lo que podría vincularse con la carencia de formación recibida y la falta de conocimiento de las implicaciones pedagógicas que esta enseñanza genera en su práctica docente.

\section{Muestra}

En este estudio participaron un total de 36 docentes (21 mujeres y 15 hombres). Concretamente, 17 impartían docencia en el Grado de Educación Primaria y 19 en el Grado de Educación Social. El contexto en el que se llevó a cabo fue la Facultad de Ciencias de la Educación de la Universidad de Málaga.

El muestreo efectuado fue de tipo teórico, por lo que la estructuración de la muestra se realizó atendiendo a la relevancia y las expectativas de los casos, y no a su representatividad. En este sentido, se solicitó la participación voluntaria de profesorado con docencia en las titulaciones mencionadas, para conocer posibles diferencias en el modo de abordar la sostenibilidad en función del Grado impartido.

Recurriendo al directorio de profesorado presente en la página Web de la Facultad de Ciencias de la Educación de la Universidad de Málaga, los contactos fueron emprendidos vía telefónica y correo electrónico para concertar día y lugar exacto en el que poder efectuar la entrevista.

Durante su proceso de realización, se tuvo en cuenta el criterio de saturación teórica (Flick, 2012), finalizando la recogida de evidencias una vez que dejaron de emerger nuevas perspectivas sobre la cuestión central objeto de estudio.

\section{Instrumento de recogida de información y tipo de análisis}

Para la recogida de información se realizaron entrevistas semiestructuradas ad hoc al profesorado universitario. Las cuestiones se redactaron por los investigadores teniendo en cuenta, tanto los objetivos del estudio, como las tendencias actuales en esta temática, y así, la fundamentación conceptual de la Educación para el Desarrollo Sostenible en el escenario universitario (Albareda et al., 2017; Andrades, Larrán y Muriel de los Reyes, 2018; Leal-Filho et al., 2017; Segalás y Sánchez-Carracedo, 2018; Serrate et al., 2019; Valderrama et al., 2020).

En concreto, las preguntas formuladas fueron las siguientes:

- ¿Considera que la universidad ha cambiado a nivel educativo y social en los últimos años? ¿cómo afecta todo ello al trabajo docente?

- ¿Debe comprometerse la universidad en la resolución de conflictos ambientales? ¿de qué forma? 
- ¿Qué relaciones podría establecer entre la universidad y la Educación para el Desarrollo Sostenible?

- ¿Qué función piensa que desempeña la Educación para el Desarrollo Sostenible en el contexto universitario?

- ¿Conoce alguna iniciativa emprendida por la Universidad de Málaga relacionada con esta enseñanza? ¿ha participado en algún momento?

- ¿Conoce líneas de investigación en la Universidad de Málaga vinculadas con la Educación para el Desarrollo Sostenible?

- ¿Piensa que en su Departamento hay compromiso para hacerla realidad?

- ¿Posee conocimientos sobre la Educación para el Desarrollo Sostenible y el concepto de sostenibilidad?

- ¿En qué medida piensa que influye todo ello en su rol profesional?

- ¿Se ha planteado, en algún momento, introducir cambios en su práctica para trabajar una educación sostenible desde las asignaturas impartidas? De ser así, ¿en qué han consistido?

- ¿Cómo piensa que podría trabajar la Educación para el Desarrollo Sostenible?

- ¿Qué competencias son necesarias para trabajar la sostenibilidad en la Universidad? ¿a qué atribuiría su posible carencia?

- ¿Ha recibido orientaciones para hacer realidad la Educación para el Desarrollo Sostenible en el contexto universitario?

- Durante su etapa de formación inicial, ¿ise contempló una educación relacionada con la sostenibilidad?

- ¿Considera que la universidad facilita la formación permanente en Educación para el Desarrollo Sostenible?

Para estudiar las valoraciones del profesorado entrevistado, se optó por la realización de un análisis de contenido de las entrevistas. Las primeras lecturas propiciaron una selección de fragmentos textuales con fundamento sustantivo, codificados para favorecer una sencilla localización de cada uno de los enunciados contemplados en la entrevista. En la codificación e identificación del lugar preciso en las transcripciones de los enunciados textuales, se recurrió al Programa Atlas.ti 8.04 (2013), como herramienta para analizar datos cualitativos, al permitir vincular códigos con fragmentos textuales, organizando y reagrupando la información sistemáticamente.

$\mathrm{Al}$ definir las categorías, primero se delimitaron dimensiones concretas, con la finalidad de construir un marco comprensivo para cada uno de los docentes de este estudio, a partir de los enunciados aportados sobre las prácticas pedagógicas fundamentadas en la Educación para el Desarrollo Sostenible. Tanto los fragmentos de texto como las evidencias literales fueron referentes durante todo el proceso de análisis de contenido, categorización, revisión de resultados y delimitación de conclusiones.

La elaboración del sistema de categorías se llevó a cabo a través de un proceso deductivo e inductivo. Deductivo, en tanto que se partió de una revisión bibliográfica y conceptual del objeto de estudio (Alba, 2017; Geli de Ciurana, Collazo y Mulá, 2019; Mulá et al., 2017; Murga-Menoyo y Novo, 2017; Valderrama et al., 2020; Villamandos, Gomera y Antúnez, 
2019). También inductivo, puesto que se debatió y se llegó a un consenso entre los investigadores para asegurar que se cumplían las condiciones necesarias con las que delimitar el sistema de categorías (Tójar, 2006).

En el cuadro 1 se recogen las dimensiones en las que se ha apoyado el estudio de los datos de la investigación y las categorías definidas. Se incluye la forma abreviada del concepto de Educación para el Desarrollo Sostenible (EDS).

Cuadro 1. Dimensiones y categorías para el análisis de fragmentos de las entrevistas

\begin{tabular}{ll}
\hline \multicolumn{1}{c}{ DIMENSIONES } & \multicolumn{1}{c}{ CATEGORÍAS } \\
\hline \multirow{2}{*}{ Sostenibilidad en el escenario universitario } & Universidad Sostenible \\
& $\begin{array}{l}\text { Funciones de la EDS } \\
\text { Iniciativas de EDS }\end{array}$ \\
\hline \multirow{2}{*}{$\begin{array}{l}\text { Desarrollo de la Educación para el Desarrollo } \\
\text { Sostenible en la universidad }\end{array}$} & $\begin{array}{l}\text { Rol del docente } \\
\text { Aplicación práctica } \\
\text { Compromiso con EDS }\end{array}$ \\
\hline $\begin{array}{ll}\text { Formación en Educación para el Desarrollo } \\
\text { Sostenible }\end{array}$ & $\begin{array}{l}\text { Formación docente } \\
\text { Aptitudes en EDS }\end{array}$ \\
\hline
\end{tabular}

Fuente: Elaboración propia.

La dimensión 1, "sostenibilidad en el escenario universitario", analiza la presencia de la sostenibilidad en la universidad, partiendo de un análisis sobre sus funciones y cambios experimentados en los últimos años, incidencia en el trabajo del profesorado y papel que, en este contexto, puede desempeñar la Educación para el Desarrollo Sostenible. En el cuadro 2 se muestra una descripción de las categorías que se integran en esta primera dimensión.

Cuadro 2. Descripción de las categorías recogidas en la primera dimensión de análisis

\begin{tabular}{cc}
\hline CATEGORÍA & \multicolumn{1}{c}{ DESCRIPCIÓN } \\
\hline Universidad & $\begin{array}{c}\text { Percepción del profesorado sobre la actual universidad, cambios destacados } \\
\text { Sostenible los últimos años, incidencia en el trabajo docente, respuesta de los } \\
\text { centros de formación superior a problemas sociales y relaciones entre } \\
\text { universidad y Educación para el Desarrollo Sostenible }\end{array}$ \\
\hline Funciones de la & $\begin{array}{l}\text { Funciones de la Educación para el Desarrollo Sostenible en el contexto } \\
\text { universitario }\end{array}$ \\
\hline EDS & $\begin{array}{l}\text { Iniciativas universitarias para impulsar la Educación para el Desarrollo } \\
\text { Sostenible, líneas de investigación relacionadas y compromiso del }\end{array}$ \\
EDS & Departamento de pertenencia con el paradigma de la sostenibilidad \\
\hline
\end{tabular}

Fuente: Elaboración propia.

La dimensión 2, "desarrollo de la Educación para el Desarrollo Sostenible en la universidad", se centra en dilucidar en qué medida el docente universitario presenta conocimientos sobre esta disciplina, reconocimiento de su incidencia en la práctica profesional, así como la responsabilidad que, a su juicio, deben asumir los centros de Educación Superior para abordar y resolver problemas ambientales. Se ofrece a continuación una delimitación de las categorías definidas que se corresponden con esta dimensión y sus aspectos fundamentales (cuadro 3). 
Cuadro 3. Descripción de las categorías recogidas en la segunda dimensión de análisis

\begin{tabular}{ll}
\hline \multicolumn{1}{c}{ CATEGORÍA } & DESCRIPCIÓN \\
\hline $\begin{array}{c}\text { Conocimientos } \\
\text { previos en EDS }\end{array}$ & $\begin{array}{c}\text { Percepciones y definición otorgada por el profesorado universitario a la } \\
\text { Educación para el Desarrollo Sostenible }\end{array}$ \\
\hline Rol del docente & $\begin{array}{c}\text { Incidencia de la Educación para el Desarrollo Sostenible en el rol del } \\
\text { docente de universidad }\end{array}$ \\
\hline Aplicación Práctica & $\begin{array}{c}\text { Puesta en práctica de la Educación para el Desarrollo Sostenible desde } \\
\text { las asignaturas impartidas y modificaciones pedagógicas relevantes } \\
\text { para hacer realidad la sostenibilidad }\end{array}$ \\
\hline $\begin{array}{c}\text { Compromiso con } \\
\text { EDS }\end{array}$ & $\begin{array}{c}\text { Universidad como escenario implicado y responsable en trabajar } \\
\text { cuestiones ambientales que lleguen a repercutir en la mejora del } \\
\text { entorno }\end{array}$ \\
\hline Fuente:
\end{tabular}

Fuente: Elaboración propia.

La tercera y última dimensión, "formación en Educación para el Desarrollo Sostenible", analiza la formación inicial y permanente del profesorado universitario en el marco de esta disciplina, directrices recibidas para su integración curricular y competencias para hacerla realidad. En el cuadro 4 se aporta una descripción de las categorías incluidas en esta tercera dimensión.

Cuadro 4. Descripción de las categorías recogidas en la tercera dimensión de análisis

\begin{tabular}{ccc}
\hline CATEGORÍA & \multicolumn{3}{c}{ DESCRIPCIÓN } \\
\hline Formación Docente & $\begin{array}{c}\text { Presencia de la Educación para el Desarrollo Sostenible en la formación } \\
\text { inicial y permanente del profesorado universitario: posibles } \\
\text { orientaciones recibidas }\end{array}$ \\
\hline Aptitudes en EDS & $\begin{array}{c}\text { Capacidades necesarias para trabajar la Educación para el Desarrollo } \\
\text { Sostenible en la universidad }\end{array}$ \\
\hline Fuente:
\end{tabular}

Fuente: Elaboración propia.

\section{Resultados}

Para exponer los resultados de esta investigación se han considerado las aportaciones más significativas de los protagonistas, en coherencia con el proceso de categorización, objetivos e hipótesis de partida.

En la dimensión "sostenibilidad en el escenario universitario", se presentan tres categorías que recogen información sobre el papel de la universidad en el marco de la Educación para el Desarrollo Sostenible, las distintas funciones a asumir e iniciativas para hacerla realidad en la formación superior.

En la categoría Universidad Sostenible, cabe mencionar que el profesorado participante en este estudio define la universidad como un espacio que debe llevar a la adquisición de competencias vitales y profesionales, argumentación sólida y cuestionamiento sobre los principales conflictos sociales, todo ello para contribuir a la transformación social desde el desempeño profesional. Perciben este espacio dinámico y abierto, en el que se construye de forma permanente el conocimiento, siendo el diálogo y el acompañamiento al alumnado algunas de sus esencias principales.

Como uno de los cambios relevantes de la universidad en las últimas décadas se hace alusión a la mayor sensibilidad hacia los conflictos sociales, lo que ha llevado a trascender prácticas magistrales tradicionales en un intento por abrir las aulas a la realidad y sus conflictos. En este sentido, admiten que la universidad debe comprometerse activamente 
en el abordaje de cuestiones vinculadas con la degradación medioambiental desde todas las titulaciones universitarias, y no solo las relacionadas con la educación, teniendo en cuenta que la crisis planetaria constituye uno de los principales problemas ante los que nos encontramos.

De la misma forma, se argumenta que, los cambios vertiginosos por los que ha transitado la universidad en los últimos años inciden en la praxis docente, en la medida en que el profesor de universidad actual tiene que hacer frente a cada vez más exigencias para consolidar su carrera profesional. Todo ello, en relación con el tema central de esta investigación, representa, bajo la óptica de los docentes entrevistados, dificultades para identificar cuál es la mejor forma de trabajar la Educación para el Desarrollo Sostenible desde las asignaturas impartidas.

Por otra parte, admiten que uno de los principales retos del docente universitario es el de propiciar la construcción de aprendizajes significativos, que lleven a desarrollar la profesión con eficacia y responsabilidad. Sumado a lo anterior, se subraya la relevancia de favorecer la promoción de estrategias que permitan la construcción y regulación autónoma de conocimientos, el pensamiento crítico y el desarrollo de competencias emprendedoras.

Con respecto a la categoría Funciones de la Educación para el Desarrollo Sostenible, los docentes reconocen que debe ser una herramienta pedagógica para educar en valores, ofrecer información sobre los problemas ambientales y sus efectos, además de sensibilizar y concienciar. Sostienen que su puesta en práctica precisa un trabajo interdisciplinar, buscando sinergias con especialistas.

En la categoría de Iniciativas de Educación para el Desarrollo Sostenible, el profesorado participante reconoce carecer de información suficiente sobre los proyectos de la Universidad de Málaga relacionados con la misma, reclamando una mayor visibilidad. Como líneas de investigación que trabajen estas cuestiones, algunos profesores aluden al Emprendimiento Sostenible, si bien admiten no liderarlas. En cuanto al grado de compromiso de los Departamentos Universitarios en proyectos ambientales, los docentes reconocen carecer de información sobre ello, aunque sostienen la necesidad de que los departamentos se impliquen en informar al alumnado sobre el nuevo campo profesional emergente gracias a esta enseñanza.

En la dimensión "desarrollo de la Educación para el Desarrollo Sostenible en la universidad", se integran cuatro categorías que recogen información sobre los conocimientos previos de los que dispone el sector docente acerca de esta disciplina, repercusión en su ejercicio profesional, posible aplicación práctica en el aula universitaria y compromiso que la universidad debe asumir en la resolución de conflictos ambientales.

En la categoría Conocimientos previos en Educación para el Desarrollo Sostenible, las entrevistas evidencian que el profesorado cuenta con un conocimiento general de la misma. Perciben que constituye un modo de educar en actitudes de respeto al medio, definiendo el Desarrollo Sostenible como una forma de educar a las generaciones del presente en el respeto al entorno.

Respecto a la categoría Rol Docente y Aplicación práctica, los docentes reconocen que la principal repercusión que genera el paradigma de la sostenibilidad en la actuación profesional se refleja en la necesidad de trabajar cuestiones vinculadas con la degradación ambiental desde las asignaturas, introduciéndolas en los bloques de contenido de forma 
transversal e identificando un nexo de unión entre este tipo de temáticas y aquellas que forman parte de las materias a trabajar. Sostienen que es necesario replantear metodologías, propiciar el protagonismo del alumnado, fomentar trabajos de investigación y coordinarse con profesionales especializados.

En el caso del profesorado con docencia en el Grado de Educación Social, define como reto a asumir la promoción de programas de intervención socioeducativa que guarden relación con la sostenibilidad. Los que imparten docencia en el Grado de Educación Primaria, por su parte, vislumbran la necesidad de hacer conscientes a los futuros maestros de Primaria la importancia de favorecer la apertura de la escuela a su entorno.

En la categoría Aplicación Práctica, ha podido evidenciarse el predominio de cierta "lógica disciplinar", en la medida en que se argumenta que para hacer realidad una educación sostenible en la universidad la opción más sencilla es recurrir a asignaturas vinculadas al ámbito de las ciencias, desde las que se puede hacer al alumnado consciente del potencial del entorno para incrementar la motivación e interés de los futuros estudiantes y colectivos con los que trabajar. Como modificaciones para que los principios de esta enseñanza estén presentes en las asignaturas, el profesorado ha reconocido la importancia del trabajo sistemático, con una previa planificación que quede delimitada en las programaciones docentes. En cuanto a la categoría de Compromiso con la Educación para el Desarrollo Sostenible, los docentes no dudan de la responsabilidad a asumir por la universidad en la resolución de problemas relacionados con el deterioro ambiental. Para ello, advierten la necesidad de organizar encuentros formativos, grupos de trabajo interuniversitarios e investigaciones.

En la dimensión "formación en Educación para el Desarrollo Sostenible", se exponen dos categorías que recogen información sobre la formación inicial y permanente del profesorado en esta disciplina, así como las habilidades necesarias para abordarla en el contexto universitario. En relación con la categoría Formación Docente, el profesorado reconoce no haber contado con una preparación explícita en cuestiones relacionadas con la sostenibilidad, si bien sostiene que, de forma tímida desde algunas asignaturas, durante el proceso de formación inicial, se ponían de relieve las ventajas del entorno para suscitar la curiosidad e interés hacia los aprendizajes. En el proceso de formación permanente, por su parte, los docentes manifiestan no haber recibido orientaciones específicas para la inclusión transversal de la Educación para el Desarrollo Sostenible en el currículum universitario, al no haber asistido a cursos formativos en esta línea ofertados por el Servicio de Personal Docente e Investigador de la Universidad de Málaga. Aun así, consideran que, más allá de una sólida preparación, es necesaria una actitud positiva para implementar cambios en el enfoque otorgado a las asignaturas, además de apertura a la innovación educativa.

Subrayan la necesidad de superar el aislamiento profesional, identificando en las reuniones de coordinación espacios apropiados para intercambiar experiencias y conocer posibles propuestas. Como aspectos a priorizar en los programas formativos, insisten en la necesidad de recibir asesoramiento en las estrategias metodológicas más idóneas para abordar la sostenibilidad, considerando que una buena opción podrían ser las experiencias de Aprendizaje Servicio y Cooperativo. Finalmente, en la categoría Aptitudes en Educación para el Desarrollo Sostenible, el profesorado alude a la relevancia de acceder a una formación continua, diversificada y coherente con la perspectiva de la sostenibilidad, siendo ello la clave para adquirir competencias profesionales en este terreno. También 
acentúan el valor del interés y compromiso, argumentando que las variables motivacionales tienen un peso determinante.

Entre algunos de los factores que, a su juicio, podrían explicar la carencia de dichas competencias, reconocen que, junto a la falta de una actitud comprometida, habría que añadir una cierta tendencia al conformismo, al trabajar bajo un mismo enfoque las asignaturas que llevan impartiéndose varios cursos académicos, sin reorientar la metodología ni velar por la integración transversal de conflictos sociales y ambientales. Argumentan que la adquisición de habilidades para hacer realidad la sostenibilidad debe ir acompañada del desarrollo de tareas autónomas de investigación, junto con el establecimiento de alianzas interuniversitarias, puesto que todo ello generará un enriquecimiento profesional y permitirá avanzar en la conformación de una universidad sostenible.

\section{Discusión y conclusiones}

Esta investigación ofrece datos de interés sobre percepciones y competencias del profesorado de la Facultad de Educación de la Universidad de Málaga en la Educación para el Desarrollo Sostenible.

Los resultados desvelan que los docentes universitarios identifican como desafíos actuales la promoción de valores y actitudes, el desarrollo de competencias para un efectivo ejercicio profesional y el abordaje de cuestiones que trasciendan lo magistral e inciten el desarrollo de conductas críticas; aspectos clave para ayudar a los estudiantes en su proceso de conformación de la identidad.

En sus argumentos se vislumbra el reconocimiento de los cambios atravesados por las universidades en los últimos años, al ser escenarios marcados por la competitividad y la presión institucional, generando incertidumbres para desarrollar la profesión. Este aspecto también es reconocido por diversos autores (Ahedo, 2016; Ruiz-Corbella y LópezGómez, 2019), quienes manifiestan la necesidad de trascender el academicismo para ofrecer una formación humanista, con la que comprender los retos del mundo actual. La universidad actual, desde las aportaciones de los docentes de este estudio y tratando de desvelar en qué medida este sector profesional advierte que esta deba comprometerse en el abordaje de la sostenibilidad, es percibida como espacio que debe abrir sus puertas a los conflictos sociales más relevantes, dado que su misión principal es formar ciudadanos responsables con el progreso social, y, con ello, implicados en la resolución de conflictos ecológicos.

Estos datos concuerdan con otras investigaciones (Aznar et al., 2017; Bautista y Díaz, 2017; Martínez-Lirola, 2018), en las que se pone de relieve que la universidad debe avanzar en el proceso de sostenibilidad curricular, al objeto de ofrecer a los estudiantes experiencias de aprendizaje con las que desarrollarse personal y laboralmente en el marco de la sostenibilidad, para replantear soluciones que den respuesta a la degradación planetaria, alentando procesos educativos fundamentados en la justicia ambiental. Reconociendo que la crisis planetaria es uno de los problemas más graves por los que atraviesa la humanidad, las evidencias de los docentes entrevistados ponen de manifiesto que, no solo defienden la importancia de configurar una universidad sostenible, sino que son conscientes de las incidencias generadas por el paradigma de la sostenibilidad en su rol profesional, lo que definen como ser capaces de promover valores y actitudes de respeto 
y defensa hacia el Planeta. En este sentido, se vislumbra que el profesorado participante en esta investigación ha apropiado el discurso de la sostenibilidad en su práctica profesional y muestra un cierto compromiso en la integración de los principios de la Educación para el Desarrollo Sostenible en sus asignaturas, aunque prevalece una "lógica disciplinar", puesto que la gran mayoría sostiene que trabajar estos aspectos resulta más sencillo cuando la relación con el medio ambiente está claramente delimitada.

Los planteamientos anteriores coinciden con otras investigaciones (Albareda et al., 2017; Litzner y Rieb, 2019; Mulá et al., 2017), en las que se argumenta que el docente universitario reconoce la necesidad de trabajar la sostenibilidad para que el alumnado asuma posturas críticas y reflexivas en torno a una cuestión que actualmente es trascendente, si bien ello afecta más directamente a asignaturas vinculadas con temas ambientales, no siendo obligatorio abordarlo en aquellas que se alejan de estos asuntos. La cuestión clave reside, a nuestro juicio, en introducir un auténtico abordaje interdisciplinar, transversal e inclusivo, y, por tanto, una promoción activa de una educación comprometida con la mejora y el respeto medioambiental en los procesos de enseñanza y aprendizaje por competencias en todas las asignaturas y áreas curriculares. Es por ello por lo que estos profesionales hacen explícita la necesidad de recibir una formación amplia, que permita el abordaje real de la sostenibilidad en la universidad.

Sumado a lo anterior, reconocen no haber recibido una formación específica sobre las repercusiones de la Educación para el Desarrollo Sostenible en el escenario universitario, de la misma forma que admiten que a lo largo de su proceso de formación inicial esta enseñanza no se trabajaba en profundidad. En este sentido, el profesorado no se define formado para trasladar el paradigma de la sostenibilidad al aula universitaria, aunque argumenta que las habilidades necesarias no dependen exclusivamente de la formación, sino que son fundamentales las variables emocionales, y, con ellas, la sensibilidad, el interés y la apertura al cambio educativo.

Estas valoraciones también se recogen en otros estudios (Escámez y López, 2019; Geli de Ciurana, Collazo y Mulá, 2019), subrayando que, para promover la sostenibilidad en el aula, es necesaria, además de una sólida formación, disponer de inquietud en la creación de situaciones que generen un aprendizaje transformador, para que el estudiante se empodere, cambie los modos de ver y pensar el mundo y llegue a su comprensión global.

En cuanto a la forma de integrar los principios de la sostenibilidad en las materias en las que tienen asignada docencia, perciben en las experiencias de Aprendizaje Servicio y de Aprendizaje Cooperativo herramientas pedagógicas beneficiosas para abordar los conflictos relacionados con el deterioro medioambiental, tratando de propiciar el desarrollo de experiencias de aprendizaje compartido a través del análisis y estudio de caso de aspectos que guarden relación con el deterioro del Planeta. Estos resultados forman también parte de otras investigaciones (Aramburuzabala, 2013; Aramburuzabala, Cerrillo y Tello, 2015; Collazo y Geli de Ciurana, 2017; Vázquez, 2015), en las que ambas estrategias se definen positivas para trabajar la sostenibilidad en el contexto universitario, en la medida en que se apoyan en una educación experiencial al servicio de la mejora de la sociedad.

$\mathrm{Al}$ establecer relaciones entre los centros de Educación Superior y el paradigma de una educación sostenible, los docentes que han formado parte de esta investigación han expresado que estas deben caracterizarse por el compromiso, necesario para diseñar actividades formativas, jornadas de sensibilización y conformación de grupos de trabajo 
que permitan el intercambio de experiencias. En la comunidad científica también hay aportaciones vinculadas a las valoraciones del profesorado de esta investigación (Aleixo, Azeiteiro y Leal, 2017; Villamandos, Gomera y Antúnez, 2019), reflejándose la necesidad de promover, junto a las actividades formativas, la organización de congresos y seminarios, con los que enriquecerse y desarrollar un trabajo compartido.

A la vista de los resultados obtenidos en este estudio, por tanto, se vislumbra que el profesorado entrevistado entiende la Educación para el Desarrollo Sostenible en la línea que plantean autores de la talla de Murga-Menoyo y Novo (2017), en tanto que son conscientes de la necesidad de recurrir a métodos participativos, experiencias de Aprendizaje Servicio, y, en definitiva, fundamentar la docencia en un enfoque pedagógico orientado a la acción y el aprendizaje transformador. No obstante, reconoce encontrarse con dificultades, no solo relacionadas con una formación escasa, sino atendiendo a las propias características de la universidad, a la que definen como institución inhóspita, que vincula la excelencia académica más con labores de investigación que de docencia y en la que al estar varios años impartiendo la misma asignatura, se generan actitudes conformistas.

En coherencia con los planteamientos expuestos a lo largo de este estudio, se reconoce la necesidad de emprender una investigación de este tipo. Aun así, debe señalarse la existencia de algunas limitaciones, en la medida en que tan solo se han recogido aportaciones de profesorado que forma parte de la Facultad de Educación de la Universidad de Málaga. Es por ello por lo que sería enriquecedor contar con la participación de profesorado universitario perteneciente a otros Grados, al objeto de establecer comparaciones en torno a la forma de hacer efectivo el paradigma de la sostenibilidad en distintas titulaciones de la Universidad de Málaga. De la misma forma, el hecho de haber optado por la realización de entrevistas como único instrumento para dar respuesta a los objetivos de este estudio, constituye otra de sus limitaciones, siendo adecuada la realización de futuras investigaciones recurriendo a técnicas de recogida de información cuantitativas, con la finalidad de llevar a cabo una triangulación de los resultados obtenidos. También sería interesante acercarse a las aportaciones del alumnado universitario, para analizar la posible formación recibida en el marco de la Educación para el Desarrollo Sostenible. En definitiva, se concluye subrayando la importancia de que la universidad asuma con seriedad el compromiso de apoyar al estudiante en la adquisición de valores y comportamientos que guiarán su conducta como ciudadano y profesional, figurando, sin duda, los de carácter ambiental.

\section{Referencias}

Ahedo, J. (2016). La universidad: Una escuela al servicio de la verdad. Revista Complutense de Educación, 27(2), 517-532. http://doi.org/10.5209/rev_RCED.2016.v27.n2.46604

Alba, D. (2017). Hacia una fundamentación de la sostenibilidad en la educación superior. Revista Iberoamericana de Educación, 73, 15-34.

Albareda, S., Fernández, M., Mallarach, J. M. y Vidal, S. (2017). Barreras para la sostenibilidad en la universidad. Revista Iberoamericana de Educación, 73, 253-272.

Aleixo, A. M., Azeiteiro, U. y Leal, S. (2017). Un decade of education for sustainable development: Perceptions of higher education institution's skateholders. En W. Leal Filho, U. M. Azeiteiro, F. Alves y P. Molltan-Hill (Eds.), Handbook of theory and practice of sustainable 
development in higher education (pp. 417-429). Springer.

https://doi.org/10-1007/978-3-319-47877-7

Andrades, F. J., Larrán, M. y Muriel de Los Reyes, M. (2018). Analysing the incorporation of sustainability themes into the university curricula: A case study of a Spanish public university. International Journal of Sustainable Development $\Xi^{2}$ World Ecology, 25(7), 642,654. https://doi.org/10.1080/13504509.2018.1437484

Antúnez, M., Gomera, A. y Villamandos, F. (2017). Sostenibilidad y currículum: Problemática y posibles soluciones en el contex to universitario español. Profesorado. Revista de Currículum y Formación de profesorado, 21(4), 197-214.

Aramburuzabala, P. (2013). Aprendizaje-servicio: Una herramienta para educar desde y para la justicia social. Revista Internacional de Educación para la Justicia Social, 2(2), 5-11.

Aramburuzabala, P., Cerrillo, R. y Tello, I. (2015). Aprendizaje-servicio: Una propuesta metodológica para la introducción de la sostenibilidad curricular en la universidad. Profesorado. Revista de Currículum y Formación del Profesorado, 19(1), 78-95.

Aznar, P., Ull, M. A., Piñero, A. y Martínez-Agut, M. P. (2014). La sostenibilidad en la formación universitaria: Desafíos y oportunidades. Educación XXI, 17(1), 133-158. https://doi.org/10.5944/educxx 1.17.1.10708

Aznar, P., Ull, M. A., Martínez-Agut, M. P. y Piñero, A. (2017). Evaluar para transformar: Evaluación de la docencia universitaria bajo el prisma de la sostenibilidad. Enseñanza de las Ciencias, 35(1), 5-27. https://doi.org/10.5565/rev/ensciencias.2112

Barth, B., Godemann, J., Rieckmann, M. y Stoltenberg, U. (2007). Developing key competencies for sustainable development in higher education. International Journal of Sustainability in Higher Education, 8(4), 416-430.

Bautista, M. J. y Díaz, M. J. (2017). La sostenibilidad en los grados universitarios: Presencia y coherencia. Teoría de la Educación, 29(1), 161-187.

http://doi.org/10.14201/teoredu291161187

Carneros, S., Murillo, F. J. y Moreno-Medina, I. (2018). Una aproximación conceptual a la educación para la justicia social y ambiental. Revista Internacional de Educación para la Justicia Social, 7(1), 17-36. https://doi.org/10.15366/riejs2018.7.1.001

Collado, J. (2017). O desenvolvimento sustentável na educação superior. Propostas biomiméticas e transdisciplinares. Revista Iberoamericana de Educación, 73, 203-224.

Collazo, L. M. y Geli de Ciurana, A. M. (2017). Avanzar en la educación para la sostenibilidad. Combinación de metodologías para trabajar el pensamiento crítico y autónomo, la reflexión y la capacidad de transformación del sistema. Revista Iberoamericana de Educación, 73, 131154.

CRUE. (2012). Directrices para la introducción de la sostenibilidad en el currículum. CRUE.

CRUE. (2018). Aportación conjunta de las universidades al plan de acción 2018-2020. CRUE.

Derr, V. (2017). Participation as a supportive framework for cultural inclusion and environmental justice. Revista Internacional de Educación para la Justicia Social, 6(1), 77-89. http://doi.org/10.15366/riejs2017.6.1.004

Escámez, J. I. y López, E. (2019). La formación del profesorado universitario para la educación en la gestión de la sostenibilidad. Publicaciones, 49, 53-62.

http://doi.org/10.30827/publicaciones.v49i1.9852

Flick, U. (2012). Introducción a la investigación cualitativa. Morata. 
Geli de Ciurana, A. M., Collazo, L. M. y Mulá, I. (2019). Contexto y evolución de la sostenibilidad en el currículum de la universidad española. Revista de Educación Ambiental y Sostenibilidad, 1(1), 1102. https://doi.org/10.25267/Rev_educ_ambient_sostenibilidad.2019.v1.i1.1102

Gil, D. y Vilches, A. (2017). Educación para la sostenibilidad y educación en derechos humanos: Dos campos que deben vincularse. Teoría de la Educación, 29(1), 79-100. https://doi.org/10.14201/teoredu201729179100

Guzmán, M. y Ortiz, L. O. (2019). El moderno Prometeo: El director escolar como líder mediador para la justicia social y el desarrollo sostenible. Revista Internacional de Educación y Justicia Social, 8(1), 63-79. https://doi.org/10.15366/riejs2019.8.1.004

Jiménez-Fontana, R. y García-González, E. (2017). Visibilidad de la educación ambiental y la educación para la sostenibilidad en las publicaciones españolas sobre educación científica. Revista Eureka sobre Enseñanza y Divulgación de las Ciencias, 14(1), 271-285. https://doi.org/10.25267/Rev_Eureka_ensen_divulg_cienc.2017.v14.i1.20

Leal Filho, W., Jim Wu, Y., Londero, L., Veiga, L., Azeiteiro, U., Caeiro, S. y da Rosa, L. R. (2017). Identifying and overcoming obstacles to the implementation of sustainable development at universities. Journal of Integrative Environmental Sciences, 14(1), 93-108. https://doi.org/10.1080/1943815X.2017.1362007

Litzner, L. I. y Rieb, W. (2019). la educación para el desarrollo sostenible en la universidad boliviana. Percepciones del profesorado. Teoría de la Educación, 31(1), 149-173. https://doi.org/10.14201/teri.19037

López, M. C., León, M. J. y Pérez, P. (2018). El enfoque por competencias en el contexto universitario español. La visión del profesorado. Revista de Investigación Educativa, 36(2), 529-545. https://doi.org/10.6018/rie.36.2.314351

Martínez-Lirola, M. (2018). La enseñanza de la justicia ambiental en el marco de la educación para el desarrollo sostenible en la universidad. Revista Internacional de Educación para la Justicia Social, 7(1), 53-68. https://doi.org/10.15366/riejs2018.7.1.003

Mulá I., Tilbury, D., Ryan, A., Mader, M., Dlouha, J., Mader, C., Benayas, J., Dlouhy, J. y Alba, D. (2017). Catalysing change for sustainability in higher education: A review of professional development initiatives for university educators. International Journal of Sustainability in Higher Education, $18(5)$, 798-820.

Murga-Menoyo, M. A. (2015). Competencias para el desarrollo sostenible: Las capacidades, actitudes y valores meta de la educación en el marco de la agenda global post-2015. Foro de Educación, 13(19), 55-83. https://doi.org/10.14516/fde.2015.013.019.004

Murga-Menoyo, M. A. (2017). Universidades en transición. Hacia una transformación institucional orientada al logro de la sostenibilidad. Revista Iberoamericana de Educación, 73, 61-84.

Murga-Menoyo, M. A. (2018). La formación de la ciudadanía en el marco de la agenda 2030 y la justicia ambiental. Revista Internacional de Educación para la Justicia Social, 7(1), 37-52. https://doi.org/10.15366/riejs2018.7.1.002

Murga-Menoyo, M. A. y Novo, M. (2017). Sostenibilidad, desarrollo "glocal” y ciudadanía planetaria. referentes de una pedagogía para el desarrollo sostenible. Teoría de la Educación, 29(1), 55-78. https://doi.org/10.14201/teoredu20172915578

Novo, M. y Murga-Menoyo, M.A. (2015). The processes of integrating sustainability in higher education curricula: A theoretical-practical experience regarding key competences and their cross-curricular incorporation into degree courses. En W. L. Filho (Ed.), Transformative approaches to sustainable development at universities (pp. 119-136). Springer.

ONU. (2015). Transformar nuestro mundo: La agenda 2030 para el desarrollo sostenible. ONU. 
París, S. (2019). Educación para la paz, creatividad atenta y desarrollo sostenible. Revista Internacional de Educación para la Justicia Social, 8(1), 27-41.

https://doi.org/10.15366/riejs2019.8.1.002

Segalás, J. y Sánchez-Carracedo, F. (2019). El proyecto EDINSOST. Formación en las Universidades españolas de profesionales como agentes de cambio para afrontar los retos de la sociedad. Revista de Educación Ambiental y Sostenibilidad, 1(1), 204-216.

https://doi.org/OOOO-0001-6954-7643

Serrate, S., Martín, J., Caballero, D. y Muñoz, J. M. (2019). Responsabilidad universitaria en la implementación de los objetivos de desarrollo sostenible. European Journal of Child Development, Education and Psychopatology, 7(2), 183-196.

https://doi.org/10.30552/ejpad.v7i2.119

Tójar, J. C. (2006). La investigación cualitativa. Comprender y actuar. La Muralla.

UNESCO. (2012). Educación para el desarrollo sostenible. Libro de consulta. UNESCO.

UNESCO. (2014). Declaración sobre la educación para el desarrollo sostenible. UNESCO.

UNESCO. (2017). Education for sustainable development goals. Learning objectives. UNESCO.

Ruiz-Corbella, M. y López-Gómez, E. (2019). La misión de la universidad en el siglo XXI: Comprender su origen para proyectar su futuro. Revista de la Educación Superior, 48(189), 119.

Valderrama, R., Alcántara, L., Sánchez-Carracedo, F., Caballero, D., Serrate, S., Gil, D., Vidal, S. y Miñano, R. (2020) ¿Forma en sostenibilidad el sistema universitario español? Visión del alumnado de cuatro universidades. Educación XX1, 23(1), 221-245.

https://doi.org/10.5944/educXX1.23420

Vázquez, V. (2015). El aprendizaje-servicio: Una estrategia para la formación de competencias en sostenibilidad. Foro de Educación, 13(19), 193-212.

https://doi.org/10.14516/fde.2015.013.019.009

Villamandos, F., Gomera, A. y Antúnez, M. (2019). Conciencia ambiental y sostenibilización curricular, dos herramientas en el camino hacia la sostenibilidad de la Universidad de Córdoba. Revista de Educación Ambiental y Sostenibilidad, 1(1), 1-19.

\section{Breve CV de los/as autores/as}

\section{María José Alcalá del Olmo Fernández}

Profesora Doctora en el Departamento de Didáctica y Organización Escolar de la Facultad de Ciencias de la Educación de la Universidad de Málaga. Tutora académica en la Universidad Nacional de Educación a Distancia (UNED). Profesora de posgrado en la Universidad Internacional Iberoamericana (UNINI). Doctora en Pedagogía por la Universidad de Málaga. Miembro del Grupo de Investigación HUM-1009: Innovación y Desarrollo Educativo Inclusivo (IdEi) de la Universidad de Málaga. ORCID ID: https://orcid.org/0000-0003-1796-3287. Email: mjalcaladelolmo@uma.es

\section{María Jesús Santos Villalba}

Doctora en Ciencias de la Educación dentro del Programa Oficial de Doctorado en Investigación e Innovación Educativa. Docente en el Departamento de Didáctica y Organización Escolar en la Universidad Internacional de la Rioja. Tutora académica en la Universidad Nacional de Educación a Distancia (UNED). Docente de posgrado en la 
Universidad Internacional Iberoamericana (UNINI). Miembro del Grupo de Investigación HUM 1009. Innovación y Desarrollo Educativo Inclusivo (IdEi) de la Universidad de Málaga. ORCID ID: https://orcid.org/O000-0001-6641-0916. Email: mariajesus.santos@unir.net

\section{Juan José Leiva}

Profesor Titular en el Departamento de Didáctica y Organización Escolar de la Facultad de Ciencias de la Educación de la Universidad de Málaga. Profesor Tutor en el Centro Asociado "María Zambrano" de la Universidad Nacional de Educación a Distancia (UNED) en Málaga. Director del Grupo de Investigación en Innovación y Desarrollo Educativo Inclusivo, IdEi, HUM-1009, y Editor de la Revista International Journal of New Education. ORCID ID: https://orcid.org/0000-0002-2857-8141. Email: juanleiva@uma.es

\section{Antonio Matas}

Profesor Titular del Departamento de Métodos de Investigación y Diagnóstico en Educación de la Facultad de Ciencias de la Educación de la Universidad de Málaga. Miembro del Grupo de Investigación HUM 1009. Innovación y Desarrollo Educativo Inclusivo. Universidad de Málaga. ORCID ID: https://orcid.org/0000-0003-1401-4932. Email: amatas@uma.es 